\title{
DIFFERENCE EQUATION METHOD FOR STATIC PROBLEM OF INFINITE DISCRETE STRUCTURES
}

\author{
Zdzislaw Pawlak, Jerzy Rakowski \\ Institute of Structural Engineering, Poznan University of Technologv, \\ ul. Piotrowo 5,60-965 Poznań, Poland.E-mail: jerzyrakowski@put.poznan.pl
}

Received 13 March 2001; accepted 05 Febr 2002

\begin{abstract}
The static problem of infinite, regular systems is considered. The structures are treated as discrete ones. The equilibrium conditions are derived using the FEM formulation. The set of infinite number of equilibrium conditions is replaced by one equivalent difference equation and solved in analytical form.
\end{abstract}

Keywords: finite difference method, infinite structures, Timoshenko beams.

\section{Introduction}

For solution of static problems by boundary element method (BEM) it is necessary to know the fundamental functions which are singular solutions for boundless systems. There are well-known fundamental functions for frequently used structures as strings, beams, plates, etc. In all these cases the infinite structures are treated as continuous ones, and the singular solutions are derived from the appropriate differential equations describing the considered problem [1]. In this paper an idea is presented that enables one to obtain fundamental solutions for discrete structures, that means the continuous ones divided into finite elements. If the structure is divided into regular mesh of identical finite elements, the set of equilibrium conditions derived by the use of the formulation of the finite element method (FEM) can be replaced by one difference equation [2]. This methodology can be extended for regular infinite discrete structures. In this case the infinite number of equilibrium conditions can also be replaced by one difference equation. The analytical solution given in a closed form for this equation can be obtained using the direct method [3] or the discrete Fourier transform formulated by Babuška [4].

In the paper the fundamental solutions of discrete Timoshenko beams and gridwork strips are derived. They can be used for static analysis of FE structures by BEM.

\section{Timoshenko beam}

Let us consider an infinite Timoshenko beam discretised by elements with equal spaced nodes (Fig 1), and loaded by concentrated forces $P_{r}$ and moments $M_{r}$.

We assume the exact element shape functions, which were derived for the 4-dof shear flexible finite element in [5] (Fig 2).

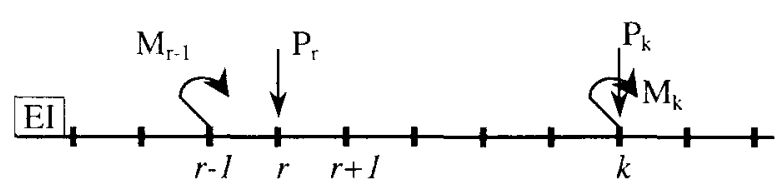

Fig 1. An infinite beam

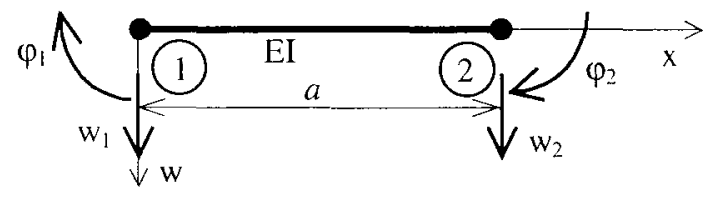

Fig 2. The finite beam element

$w(\xi)=\left[\mathbf{N}_{1}\right]^{T} q ; \phi(\xi)=\left[\mathbf{N}_{2}\right]^{T} q ; \quad \gamma(\xi)=\left[\mathrm{N}_{3}\right]^{T} q$

where: $\xi=x / a, \quad d=\frac{12 E I}{\kappa G A a^{2}}, \quad D=1 /(d+1)$,

$$
\left[\mathbf{N}_{1}\right]=\left[\begin{array}{c}
D\left[2 \xi^{3}-3 \xi^{2}-d \xi+d+1\right] \\
D\left[2 \xi^{3}-(4+d) \xi^{2}+(d+2) \xi\right] \\
D\left[-2 \xi^{3}+3 \xi^{2}+d \xi\right] \\
D\left[2 \xi^{3}-(2-d) \xi^{2}-d \xi\right] / 2
\end{array}\right]
$$

$\left[\mathbf{N}_{2}\right]=\left[\begin{array}{c}6 D\left[\xi^{2}-\xi\right] a \\ D\left[3 \xi^{2}-(d+4) \xi+d+1\right] a \\ 6 D\left[-\xi^{2}+\xi\right] a \\ D\left[3 \xi^{2}-(2-d) \xi\right] a\end{array}\right], \quad\left[\mathbf{N}_{3}\right]=\left[\begin{array}{c}-D d / 2 \\ -D d / 2 \\ D d / 2 \\ -D d / 2\end{array}\right]$ 
$E, G$ are Young's and Kirchhoff's moduli, $I$ is the moment of inertia, $A$ is the area of the beam cross-section, $\kappa$ is the shear factor.

From the expression of strain energy

$$
U=\int_{0}^{a} \frac{E I}{2}\left[\frac{d \phi}{d x}\right]^{2} d x+\int_{0}^{a} \frac{\kappa G A}{2}\left[\frac{d w}{d x}-\phi\right]^{2} d x
$$

the element stiffness matrix is determined:

$$
\begin{gathered}
{[\mathrm{K}]=\begin{array}{ll}
E I & 1 \\
a^{3} & d+1
\end{array}\left[\mathbf{K}_{\mathbf{1}}\right]+d\left[\mathbf{K}_{2}\right],} \\
{\left[\mathbf{K}_{\mathbf{1}}\right]=\left[\begin{array}{cccc}
12 & 6 & -12 & 6 \\
6 & 4 & -6 & 2 \\
-12 & -6 & 12 & -6 \\
6 & 2 & -6 & 4
\end{array}\right],} \\
{\left[\mathbf{K}_{2}\right]=\left[\begin{array}{cccc}
0 & 0 & 0 & 0 \\
0 & 1 & 0 & -1 \\
0 & 0 & 0 & 0 \\
0 & -1 & 0 & 1
\end{array}\right]}
\end{gathered}
$$

The stiffness matrix (3) refers to the element relationship:

$$
[\mathrm{K}] \mathbf{q}=\mathbf{f},
$$

where $\quad \mathbf{f}^{T}=\left\{P_{1}, m_{1}, P_{2}, m_{2}\right\}, \quad \mathbf{q}^{T}=\left\{w_{1}, \phi_{1}, w_{2}, \phi_{2}\right\}$, $\phi_{i}=a \varphi_{i}, \quad m_{i}=M_{i} / a . w_{i}$ and $\varphi_{i}$ are nodal transverse displacements and total rotations.

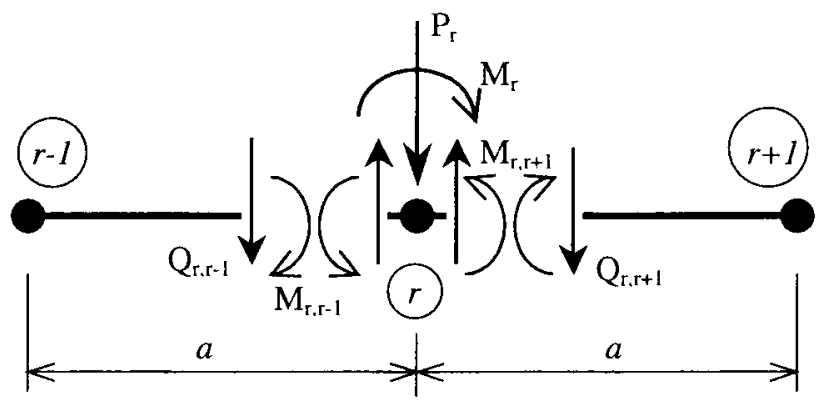

Fig 3. The internal forces at node $r$

Assembling two adjacent elements $(r-1, r)$ and $(r, r+1)$ (Fig 3) yields the following equilibrium conditions for an arbitrary beam node $r$ subjected to the nodal force $P_{r}$ and moment $M_{r}$ :

$$
\begin{aligned}
& 6\left(w_{r-1}-w_{r}\right)+2\left(\phi_{r-1}+2 \phi_{r}\right)+ \\
& +6\left(w_{r}-w_{r+1}\right)+2\left(2 \phi_{r}+\phi_{r+1}\right)+ \\
& +d\left(-\phi_{r-1}+\phi_{r}\right)+d\left(\phi_{r}-\phi_{r+1}\right)=\frac{a^{3}}{E I}(d+1) m_{r}
\end{aligned}
$$

$$
\begin{aligned}
& -12\left(w_{r-1}-w_{r}\right)-6\left(\phi_{r-1}+\phi_{r}\right)+ \\
& +12\left(w_{r}-w_{r+1}\right)+6\left(\phi_{r}+\phi_{r+1}\right)={ }_{E I}^{a^{3}} P_{r} .
\end{aligned}
$$

Introducing in (5) the shifting operator $E_{f}$. $=E^{n}$ and central difference operator $\Delta_{r}^{2}=\Delta^{2}=E+E^{-1}-2$, we obtain two difference equations with unknowns $\phi_{r}$ and $w_{r}$ :

$$
\begin{gathered}
{\left[\left(\Delta^{2}+6\right)-\frac{d}{2} \Delta^{2}\right] \phi_{r}-3\left(E-E^{-1}\right) w_{r}=\frac{a^{3}}{2 E I}(d+1) m_{r},} \\
\left(E-E^{-1}\right) \phi_{r}-2 \Delta^{2} w_{r}=\frac{a^{3}}{6 E I}(d+1) P_{r} .
\end{gathered}
$$

After elimination of $\phi_{r}$. in (6) we get one fourthorder difference equation with one unknown $w_{r}$ :

$$
\Delta^{4} w_{r}=4 \mu\left(\Delta^{2}+6-\frac{d}{2} \Delta^{2}\right) P_{r}-12 \mu\left(E-E^{-1}\right) m_{r}
$$

where $\mu=a^{3} / 24 E I$.

Let us find the solution of (7) for the beam loaded by one force concentrated at the node $p\left(P_{r}=P \cdot \delta_{r, p}\right)$ and one moment concentrated at the node $m$ $\left(M_{r}=M \cdot \delta_{r, m}\right)-$ is Kronecker's delta.

Having introduced the solution of the homogeneous equation $\left(\Delta^{4} w_{r}=0\right)$ in the form

$$
w_{r}=c_{0}+c_{1} r+c_{2} r^{2}+c_{3} r^{3}
$$

we consider the equilibrium conditions of nodes $p$ and $m$. It leads to the definition of displacement discrete functions $w_{r}$ and $\phi_{r}$, which fulfil equations (6):

$$
\begin{aligned}
& w_{r}(p, m)=\mu P\left[2 r-p^{3}-d r-p \mid\right]+ \\
& +6 \mu \frac{M}{a}\left[\left(1-\frac{d}{2}\right)(r-m)-(r-m)^{2} \operatorname{sgn}(r-m)+\right. \\
& \left.+(r-m)^{3}\right], \\
& \phi_{r}(p, m)=6 \mu P(r-p)^{2} \operatorname{sgn}(r-p)+ \\
& \quad+6 \mu \frac{M}{a}\left[1-2 r-m+3(r-m)^{2}\right] .
\end{aligned}
$$

These functions can be treated as the fundamental solutions for an infinity discrete Timoshenko beam (assuming $d=0$ yields the result for Euler-Bernoulli beam).

\section{Gridwork strip}

Now let us consider the 2D gridwork strip which consists of two sets of intersecting beams. The beams of the length $S \cdot a_{y}$ in y-direction are simply supported on their ends, the set of the beams is infinite in x-direction (Fig 4). 


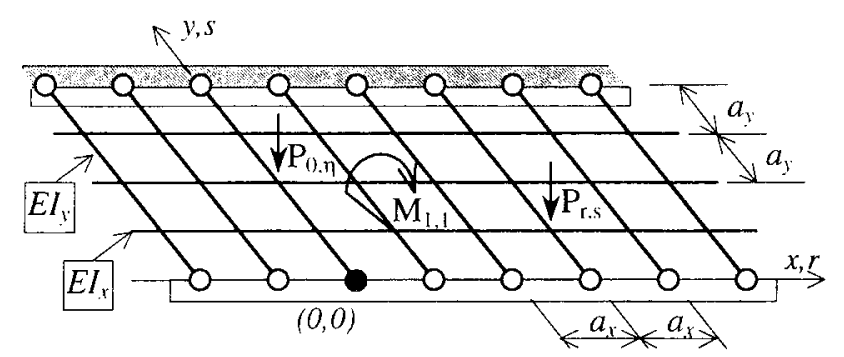

Fig 4. The infinite gridwork strip

Assuming the regular mesh of considered strip, we can derive the equilibrium conditions for each node $(r, s)$ subjected by concentrated force $P_{r, s}$ and moments $M_{r, s}^{x}$, $M_{r, s}^{y}$. Having assembled four 6-dof beam finite elements (Fig 5),

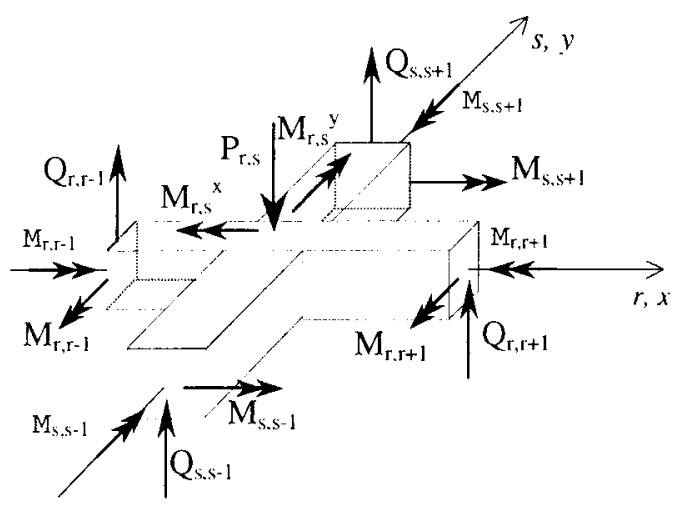

Fig 5. The gridwork node $(r, s)$

we get three difference equations equivalent to the FEM matrix formulation:

$$
\begin{aligned}
& \frac{E I_{x}}{a_{x}^{3}}\left[12 \Delta_{r}^{2} w_{r, s}+6\left(E_{r}^{-1}-E_{r}\right) \phi_{r, s}^{x}\right]+ \\
& +\frac{E I_{y}}{a_{y}^{3}}\left[12 \Delta_{s}^{2} w_{r, s}+6\left(E_{s}^{-1}-E_{s}\right) \phi_{r, s}^{y}\right]=-P_{r, s} \\
& \quad E I_{x}\left[6\left(E_{r}^{-1}-E_{r}\right) w_{r, s}+2\left(6+\Delta_{r}^{2}\right) \phi_{r, s}^{x}\right]+ \\
& \quad a_{x}^{3} \\
& +G I_{s}^{y}\left[\left(-\Delta_{s}^{2}\right) \phi_{r, s}^{x}\right]=M_{r, s}^{x} \\
& \quad a_{y} \\
& \quad E I_{y}\left[6\left(E_{s}^{-1}-E_{s}\right) w_{r, s}+2\left(6+\Delta_{s}^{2}\right) \phi_{r, s}^{y}\right]+ \\
& a_{y}^{3} \\
& +\quad G I_{s}^{x}\left[\left(-\Delta_{r}^{2}\right) \phi_{r . s}^{y}\right]=M_{r, s}^{y}, \\
& \quad a_{x}
\end{aligned}
$$

where $a_{x}, a_{y}$ are the distances between nodes in $\mathrm{x}$ and $y$-directions, respectively, $E I_{x}, G I_{s}^{x}, E I_{y}, G I_{s}^{y}$ are the bending and torsional rigidity of beams in both directions.
After elimination of rotations $\phi_{r, s}^{x}, \phi_{r, s}^{y}$ in (10) we obtain one six-order partial difference equation with one unknown $w_{r, s}$ (nodal transverse displacement):

$$
\begin{aligned}
& {\left[\Delta_{r}^{2}\left(\Delta_{s}^{2}+6-\lambda_{y} \Delta_{r}^{2}\right)\left(\Delta_{r}^{2}+2 \cdot \lambda_{x} \Delta_{s}^{2}\right)+\right.} \\
& \left.+\mu \Delta_{s}^{2}\left(\Delta_{r}^{2}+6-\lambda_{x} \Delta_{s}^{2}\right)\left(\Delta_{s}^{2}+2 \cdot \lambda_{y} \Delta_{r}^{2}\right)\right] w_{r, s}= \\
& =4 \mu_{x}\left[\left(\Delta_{r}^{2}+6-\lambda_{x} \Delta_{s}^{2}\right)\left(\Delta_{s}^{2}+6-\lambda_{y} \Delta_{r}^{2}\right) P_{r, s}+\right. \\
& \quad-3\left(E_{r}-E_{r}^{-1}\right)\left(\Delta_{s}^{2}+6-\lambda_{y} \Delta_{r}^{2}\right) M_{r, s}^{x}+ \\
& \left.\quad-3\left(E_{s}-E_{s}^{-1}\right)\left(\Delta_{r}^{2}+6-\lambda_{x} \Delta_{s}^{2}\right) M_{r, s}^{y}\right]
\end{aligned}
$$

where $\mu=\frac{\mu_{x}}{\mu_{y}} \cdot \mu_{x}=\frac{a_{x}^{3}}{24 E I_{x}}, \mu_{y}=\frac{a_{y}^{3}}{24 E I_{y}}$,

$$
\lambda_{x}=\frac{G I_{s}^{y} a_{x}^{3}}{2 E I_{x} a_{y}}, \quad \lambda_{y}=\frac{G I_{s}^{x} a_{y}^{3}}{2 E I_{y} a_{x}} .
$$

We derive the fundamental solution for the strip loaded by force $P_{0, \eta}=P_{r, s} \delta_{r, 0} \delta_{s, \eta}=P \cdot \delta_{r, 0} \delta_{s, \eta}$ $\left(M_{r, s}^{x}=M_{r, s}^{y}=0\right)$. In order to solve this problem, we use the discrete Fourier transform in $\mathrm{x}$-direction [4]:

$$
\begin{aligned}
& F\left[f_{r}\right]=\widetilde{f}(\alpha)=\sum_{r=-\infty}^{\infty} f_{r} e^{i r \alpha} \\
& F^{-1}[\tilde{f}(\alpha)]=f_{r}=\frac{1}{2 \pi} \int_{-\pi}^{\pi} \tilde{f}(\alpha) e^{-i r \alpha} d \alpha
\end{aligned}
$$

and the eigenfunction transformation in $y$-direction:

$$
w_{r, s}=\sqrt{\frac{2}{S}} \sum_{l=1}^{S-1} \widetilde{w}_{r, l} \sin \left(\frac{l \pi s}{S}\right) .
$$

Applying both transforms (12) and (13) to equation (11) we obtain the formula:

$$
W_{r, s}(0, \eta)=\frac{4 \mu_{x}}{S \pi} P \sum_{l=1}^{S-1} N_{r}^{S}(l) \cdot \sin (\varepsilon \cdot \eta) \cdot \sin (\varepsilon \cdot s),
$$

where $\varepsilon=\frac{l \pi}{S}$,

$$
\begin{gathered}
N_{r}^{S}(l)=\int_{0}^{\pi} L^{S} \cdot \cos (r \alpha) d \alpha \\
L^{S}=\left[\cos (\alpha)+2-\lambda_{x}(\cos (\varepsilon)-1)\right] \\
\cdot\left[\cos (\varepsilon)+2-\lambda_{y}(\cos (\alpha)-1)\right]
\end{gathered}
$$

$$
\begin{aligned}
M^{S} & =(\cos (\alpha)-1)\left[\cos (\alpha)-1+2 \lambda_{x}(\cos (\varepsilon)-1)\right] \\
& \cdot\left[\cos (\varepsilon)+2-\lambda_{y}(\cos (\alpha)-1)\right]+ \\
+ & \mu(\cos (\varepsilon)-1)\left[\cos (\varepsilon)-1+2 \lambda_{y}(\cos (\alpha)-1)\right] . \\
& \cdot\left[\cos (\alpha)+2-\lambda_{x}(\cos (\varepsilon)-1)\right] .
\end{aligned}
$$


The integer (15) can be calculated analytically. After some transformation we get:

$$
N_{r}^{S}(l)=\left[F_{0}(r)+S 1_{S} \cdot F_{1}(r)+S 2_{S} \cdot F_{2}(r)\right]
$$

$$
\begin{aligned}
F_{0}(r) & =2^{r-1} C(r+2)-\left(\begin{array}{l}
r \\
1
\end{array}\right) 2^{r-3} C(r)+ \\
& +\begin{array}{r}
r \\
2
\end{array}\left(\begin{array}{c}
r-3 \\
1
\end{array}\right) 2^{r-5} C(r-2)-\frac{r}{3}\left(\begin{array}{c}
r-4 \\
2
\end{array}\right) 2^{r-7} C(r-4)+\ldots
\end{aligned}
$$$$
F_{1}(r)=2^{r-1} C(r+1)-\left(\begin{array}{l}
r \\
1
\end{array}\right) 2^{r-3} C(r-1)+
$$$$
+\begin{gathered}
r \\
2
\end{gathered}\left(\begin{array}{c}
r-3 \\
1
\end{array}\right) 2^{r-5} C(r-3)-\frac{r}{3}\left(\begin{array}{c}
r-4 \\
2
\end{array}\right) 2^{r-7} C(r-5)+\ldots
$$

$$
\begin{aligned}
F_{2}(r) & =2^{r-1} C(r)-\left(\begin{array}{l}
r \\
1
\end{array}\right) 2^{r-3} C(r-2)+ \\
& +\frac{r}{2}\left(\begin{array}{c}
r-3 \\
1
\end{array}\right) 2^{r-5} C(r-4)-\frac{r}{3}\left(\begin{array}{c}
r-4 \\
2
\end{array}\right) 2^{r-7} C(r-6)+\ldots
\end{aligned}
$$

$$
S 1_{S}=1-\frac{\cos (\varepsilon)+2}{\lambda_{y}}-\lambda_{x}(\cos (\varepsilon)-1)
$$

$$
\begin{aligned}
S 2_{S} & =-2+\lambda_{x}(\cos (\varepsilon)-1)+ \\
& -\frac{\cos (\varepsilon)+2}{\lambda_{y}}\left[-2+\lambda_{x}(\cos (\varepsilon)-1)\right]
\end{aligned}
$$

where

$$
\begin{aligned}
C(n) & =\int_{0}^{\pi} \frac{\cos ^{n}(\alpha)}{\cos ^{3}(\alpha)+a_{m} \cos ^{2}(\alpha)+b_{m} \cos (\alpha)+c_{m}} d \alpha(17) \\
a_{m} & =2 \lambda_{x}(\cos (\varepsilon)-1)-[3+2 \mu(\cos (\varepsilon)-1)]-\frac{\cos (\varepsilon)+2}{\lambda_{y}} \\
b_{m}= & 3-2 \mu(\cos (\varepsilon)-1)+\frac{-3 \cos ^{2}(\varepsilon)+2 \cos (\varepsilon)+7}{\lambda_{y}} \\
& +2 \mu \frac{(\cos (\varepsilon)-1)(\cos (\varepsilon)+2)}{\lambda_{y}}+\lambda_{x}\left[2 \mu(\cos (\varepsilon)-1)^{2}+\right. \\
& \left.-4(\cos (\varepsilon)-1)-2 \frac{(\cos (\varepsilon)-1)(\cos (\varepsilon)+2)}{\lambda_{y}}\right]
\end{aligned}
$$$$
c_{m}=-1+4 \mu(\cos (\varepsilon)-1)+\frac{-6 \cos ^{2}(\varepsilon)-\cos (\varepsilon)+4}{\lambda_{y}}
$$$$
+4 \mu \frac{(\cos (\varepsilon)-1)(\cos (\varepsilon)+2)}{\lambda_{y}}+\lambda_{x}\left[-2 \mu(\cos (\varepsilon)-1)^{2}+\right.
$$$$
+2(\cos (\varepsilon)-1)-2 \mu \frac{(\cos (\varepsilon)-1)^{2}(\cos (\varepsilon)+2)}{\lambda_{y}}+
$$$$
\left.+\frac{\left(3 \cos (\varepsilon)^{2}+2 \cos (\varepsilon)+1\right)(\cos (\varepsilon)-1)}{\lambda_{y}}\right] \text {. }
$$

For (17) the following recurrent relationship is valid $(n \geq 3, k=2,3,4, \ldots)$ :

$$
\begin{aligned}
& \text { for } n=2 \cdot k-1: \quad C(n)=0 \\
& \text { for } n=2 \cdot k: \\
& \qquad \begin{aligned}
C(n) & =\pi\left\{\begin{array}{rr}
(-1)^{n-3}+1 & (n-3) ! \\
2^{n-2} & {[(0,5 n-1,5) !]^{2}}
\end{array}\right\}+ \\
& \quad-a_{m} \cdot C(n-1)-b_{m} \cdot C(n-2)-c_{m} \cdot C(n-3)
\end{aligned}
\end{aligned}
$$

The values of integral (17) for $n=0,1,2$ are as follows:

$$
\begin{aligned}
& C(n=0)=c \operatorname{sgn}\left(\frac{\left(1+m z_{1}\right)}{\sqrt{m z_{1}^{2}-1}}\right) \frac{-\pi}{a_{11} \sqrt{m z_{1}^{2}-1}}+ \\
& +\operatorname{csgn}\left(\frac{\left(1+m z_{2}\right)}{\sqrt{m z_{2}^{2}-1}}\right) \frac{-\pi}{a_{12} \sqrt{m z_{2}^{2}-1}}+ \\
& +\operatorname{sgn}\left(\frac{\left(1+m z_{3}\right)}{\sqrt{m z_{3}^{2}-1}}\right) \frac{-\pi}{a_{13} \sqrt{m z_{3}^{2}-1}} \\
& C(n=1)=\operatorname{csgn}\left(\frac{\left(1+m z_{1}\right)}{\sqrt{m z_{1}^{2}-1}}\right) \frac{-\pi \cdot m z_{1}}{a_{11} \sqrt{m z_{1}^{2}-1}}+ \\
& +c \operatorname{sgn}\left(\frac{\left(1+m z_{2}\right)}{\sqrt{m z_{2}^{2}-1}}\right) \frac{-\pi \cdot m z_{2}}{a_{12} \sqrt{m z_{2}^{2}-1}}+ \\
& +c \operatorname{sgn}\left(\frac{\left(1+m z_{3}\right)}{\sqrt{m z_{3}^{2}-1}}\right) \frac{-\pi \cdot m z_{3}}{a_{13} \sqrt{m z_{3}^{2}-1}} \\
& C(n=2)=\operatorname{csgn}\left(\frac{\left(1+m z_{1}\right)}{\sqrt{m z_{1}^{2}-1}}\right) \frac{-\pi \cdot m z_{1}^{2}}{a_{11} \sqrt{m z_{1}^{2}-1}}+ \\
& +c \operatorname{sgn}\left(\frac{\left(1+m z_{2}\right)}{\sqrt{m z_{2}^{2}-1}}\right) \frac{-\pi \cdot m z_{2}^{2}}{a_{12} \sqrt{m z_{2}^{2}-1}}+ \\
& +c \operatorname{sgn}\left(\frac{\left(1+m z_{3}\right)}{\sqrt{m z_{3}^{2}-1}}\right) \frac{-\pi \cdot m z_{3}^{2}}{a_{13} \sqrt{m z_{3}^{2}-1}} \\
& a_{11}=\left(m z_{1}-m z_{2}\right)\left(m z_{1}-m z_{3}\right), \\
& a_{12}=\left(m z_{1}-m z_{2}\right)\left(m z_{3}-m z_{2}\right) \text {, } \\
& a_{13}=\left(m z_{1}-m z_{3}\right)\left(m z_{2}-m z_{3}\right) \text {, } \\
& m z_{1}=\frac{-\Psi}{12}+\frac{\left(3 b_{m}-a_{m}^{2}\right)}{3 \Psi}-\frac{a_{m}}{3}+\frac{i \sqrt{3}}{2}\left[\frac{\Psi}{6}+\frac{\left(6 b_{m}-2 a_{m}^{2}\right)}{3 \Psi}\right] \\
& m z_{2}=\frac{-\Psi}{12}+\frac{\left(3 b_{m}-a_{m}^{2}\right)}{3 \Psi}-\frac{a_{m}}{3}-\frac{i \sqrt{3}}{2}\left[\frac{\Psi}{6}+\frac{\left(6 b_{m}-2 a_{m}^{2}\right)}{3 \Psi}\right]
\end{aligned}
$$




$$
\begin{gathered}
m z_{3}=\frac{\Psi}{6}-\frac{\left(6 b_{m}-2 a_{m}^{2}\right)}{3 \Psi}-\frac{a_{m}}{3} \\
\Psi=\left[36 a_{m} b_{m}-108 c_{m}-8 a_{m}^{3}+12 \cdot \Omega\right]^{(1 / 3)} \\
\Omega=\sqrt{\left(12 b_{m}-3 a_{m}^{2}\right)\left(b_{m}^{2}-4 a_{m} c_{m}\right)-3 c_{m}\left(2 a_{m} b_{m}-27 c_{m}\right)} .
\end{gathered}
$$

The formulas (19) include function $c \operatorname{sgn}(x)$ which determines the sign of complex number:

$$
c \operatorname{sgn}(x)=\left\{\begin{array}{cll}
1 & \text { if } & \operatorname{Re}(x)>0 \cup \operatorname{Re}(x)=0 \cap \operatorname{Im}(x)>0 \\
-1 & \text { if } & \operatorname{Re}(x)<0 \cup \operatorname{Re}(x)=0 \cap \operatorname{Im}(x)<0 .
\end{array}\right.
$$

All remaining values of unknown displacements $w_{r, s}$ are calculated from the simple recurrent relationship given in (18). The considered discrete structure can be treated as an approximation of a plate strip using the beam analogy.

\section{Numerical example}

The computations were performed for the infinite gridwork strip of wide $S_{d}=5 a$ loaded by unite force at point $(0,1)$. The results of numerical calculations are presented in Table. The plot of strip deformation is shown in Fig 6.

The displacements $W_{r, s}(0,1) \cdot E I_{x}$ of infinite strip

\begin{tabular}{|c|c|c|c|c|c|}
\hline & $r=0$ & $r=1$ & $r=2$ & $r=3$ & $r=4$ \\
\hline$s=4$ & 0,09655 & 0,08963 & 0,07296 & 0,05428 & 0,03833 \\
\hline$s=3$ & 0,17767 & 0,16244 & 0,12792 & 0,09250 & 0,06411 \\
\hline$s=2$ & 0,22433 & 0,19526 & 0,14349 & 0,09913 & 0,06694 \\
\hline$s=1$ & 0,19251 & 0,14740 & 0,09837 & 0,06503 & 0,04292 \\
\hline
\end{tabular}

The fundamental solution derived in Section 3 can be used to solve the static problem of finite gridworks in analogous way as in the boundary element method for continuous systems.

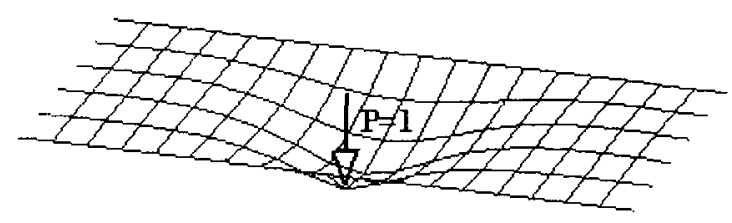

Fig 6. The infinite gridwork strip deformation

Let us consider the isotropic gridwork consisting of the set of $4 \times 4$ identical intersecting perpendicular beams (Fig 7).

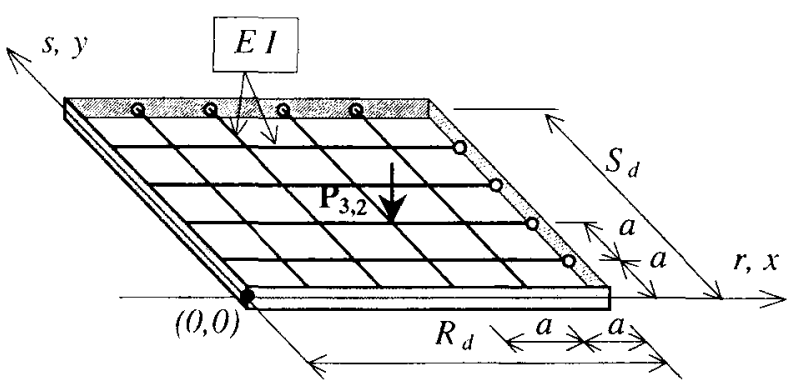

Fig 7. The isotropic gridwork system

The nodes are regular spaced in both directions of distance a. All beams are simply supported on their ends. We introduce to the given force $P_{r, s}=1 \cdot \delta_{r, 3} \cdot \delta_{s, 2}$ in the infinite strip additional loading $X_{k, j}$, in order to fulfil the required boundary conditions in the nodes lying on the beams $r=0$ and $r=5$ (Fig 8). The values of the additional forces $X_{k, j}$ are determined by the following equations (indirect method of BEM):

$$
\begin{gathered}
w_{0, i}\left(P_{r, s}, X_{k, j}\right)=0, \\
M_{0, i}\left(P_{r, s}, X_{k, j}\right)=0, \\
w_{5, i}\left(P_{r, s}, X_{k, j}\right)=0, \\
M_{5, i}\left(P_{r, s}, X_{k, j}\right)=0,
\end{gathered}
$$

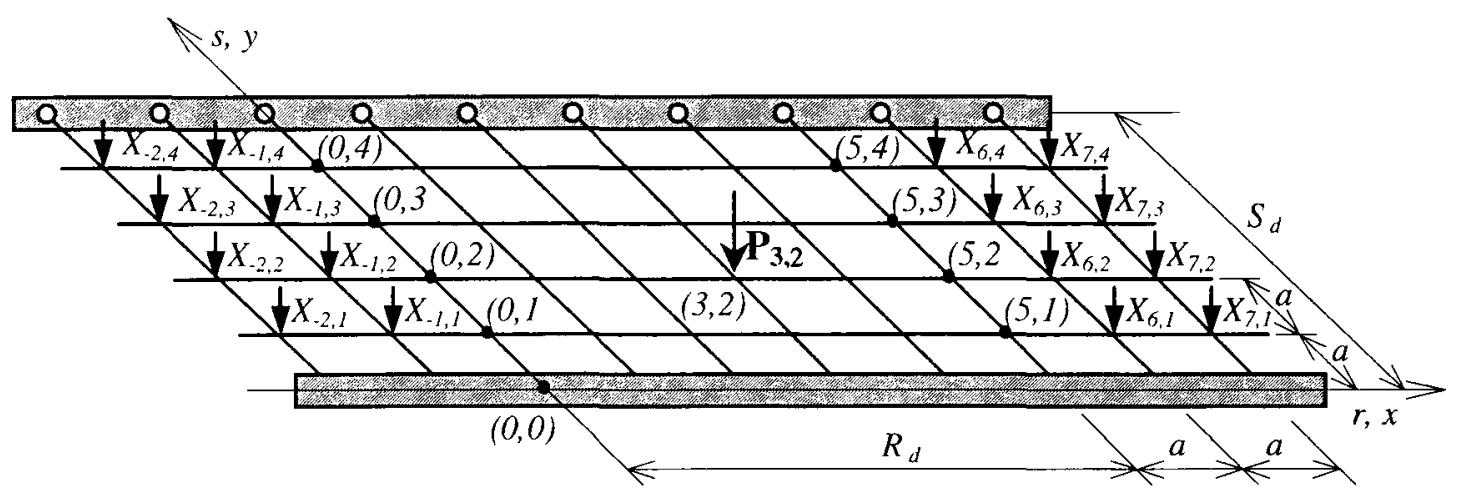

Fig 8. Additional forces applied to the infinite gridwork strip 
Having found from (20) the forces $X_{k, j}$, we get finally the displacements of finite gridwork:

$$
w_{r, s}=\sum_{k} \sum_{j} X_{k, j} W_{r, s}(k, j)+P_{3,2} W_{r, s}(3,2)
$$

where $W_{r, s}(k, j)$ is the fundamental solution calculated by (14).

The calculations are carried out for the following dimensionless parameters: $E=1, v=\frac{1}{3}, G=\frac{3}{8}, a=1$, the beam cross-section: $b \times h=1 \times 0,1$. The plot of gridwork displacement is presented in Fig 9.

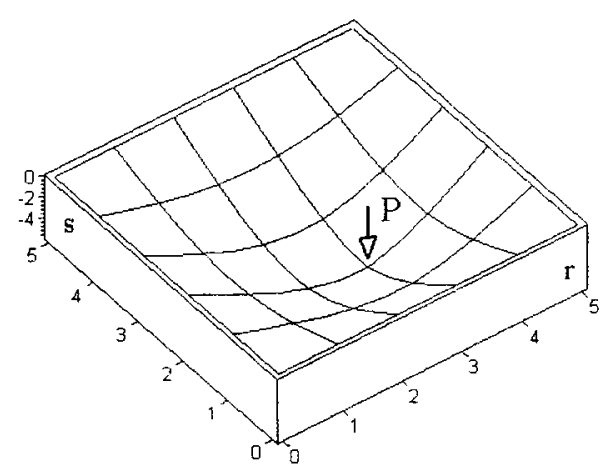

Fig 9. The displacement plot of finite gridwork system

The results coincide with ones obtained for an adequate plate strip. The relation between displacements for plate $w^{(P)}$ and gridwork $w^{(G)}$ at point $(2,3)$ subjected by unit force is $w_{3,2}^{(P)} / w_{3,2}^{(G)}=1,0018$.

\section{Conclusions}

The one- and two dimensional infinite beam systems are analysed using the finite element methodology. The infinite number of equilibrium conditions was replaced by one equivalent difference equation. The solution of these equations derived for infinite Timoshenko beam and gridwork strip are the fundamental functions for both kinds of the considered structures. They can be applied in BEM for the continuous structures discretised by finite elements.

\section{References}

1. Banerjee P. K., Butterfield R. Boundary Element Method in Engineering Science. Mc Graw-Hill, Maidenhead, 1981.

2. Rakowski J., Switka R. On some generalisations of the eigenfunction method used to the elastic discrete systems. Monograph. No 116. Politechnika Poznańska, Poznań, 1980. 92 p. (in Polish).

3. Rakowski J., Wielentejczyk P. Vibrations of infinite periodic beams by finite element method. Journal of Applied Math. and Mech. (Zeit. f. Angew. Math. u. Mech.), 76, 1996, p. 411-412.

4. Babuška I. The Fourier transform in the theory of differences equation and its applications. Arch. Applied Mech. (Arch. Mech. Stos.) 4, 11 (1959).

5. Rakowski J. The interpretation of the shear locking in beam elements. Comp. Structures, 37 (1990), p. 769-776. 